\title{
Effet des durées de conservation et du traitement à l'eau sur la germination des graines de Tieghemella heckelii Pierre ex A. Chev. (Sapotaceae) en Côte d'Ivoire
}

\author{
Kouassi KOUADIO ${ }^{*}$, Souleymane $\mathrm{SANOGO}^{2}$, Edouard Konan KOUASSI ${ }^{1}$ et \\ Cybèle Sopie DOFFOU ${ }^{1}$
}

\author{
${ }^{1}$ Laboratoire de Botanique et de Biologie Végétale, UFR Biosciences, Université Félix Houphouët-Boigny, 22 \\ BP 582 Abidjan 22, Côte d'Ivoire. \\ ${ }^{2}$ Laboratoire de Physiologie Végétale, UFR Biosciences, Université Félix Houphouët-Boigny, 22 BP 582 \\ Abidjan 22, Côte d'Ivoire. \\ *Auteur correspondant ; E-mail : attowoula@yahoo.fr; Tél. (+225) 40-13-28-03/47-90-82-75
}

\section{REMERCIEMENTS}

Nous adressons nos remerciements au Centre d'Excellence Africain sur le Changement Climatique, la Biodiversité et l'Agriculture Durable (CEA-CCBAD) de nous avoir accordé une bourse pour la réalisation de cette étude.

\section{RESUME}

Le Makoré est une espèce de bois d'œuvre de grande valeur commerciale, en danger de disparition dans les forêts tropicales. Cependant, la germination des graines fraiches et celles séchées et conservées est peu connue par l'homme. C'est pourquoi, cette étude vise à maitriser les paramètres de germination des graines de Makoré, afin de l'utiliser dans les reboisements des forêts classées en Côte d'Ivoire, en vue de sa pérennisation. Spécifiquement, il a été question de déterminer le délai de germination, la durée de germination et le taux de germination des graines. Pour ce faire, 5 lots de graines ont été constitués en fonction des durées de conservation sur lesquels 4 traitements à l'eau de robinet ont été appliqués. Les résultats ont montré que le lot 1 ou témoin (graines fraiches), le lot 2 (graines conservées pendant 14 jours) et le lot 3 (graines conservées pendant 21 jours) ont présenté des taux de germination, respectivement, de l'ordre de 72, 22, 55,56 et 41,67\%. Le trempage des graines dans l'eau de robinet a réduit considérablement le délai de germination des graines du lot 1 à 25 jours et a relevé le taux de germination du prétraitement 4 du lot 1 (graines ayant séjourné dans l'eau pendant 6 jours) à $100 \%$. Il ressort de cette étude que pour produire des plants de Makoré, les graines fraiches et celles séchées et conservées pendant deux et trois semaines peuvent être utilisées.

(C) 2019 International Formulae Group. All rights reserved.

Mots clés: Makoré, espèce menacée, conservation des graines, prétraitement, paramètres de germination, Côte d'Ivoire. 


\title{
Effect of storage times and water treatment on germination of the seeds of Tieghemella heckelii Pierre ex A. Chev. (Sapotaceae) in Côte d'Ivoire
}

\begin{abstract}
Makoré is a timber species of great commercial value, in danger of disappearing in tropical forests. However, the germination of fresh seeds and those dried and preserved is little known by man. That is why, this study aims to control the seeds germination parameters of Makoré, in order to use it in the reforestation of the classified forests in Côte d'Ivoire, with a view to its perpetuation. Specifically, there has been talk of determining germination time limit, germination duration and seed germination rate. To do this, 5 lots of seeds were constituted according to the storage periods on which 4 treatments with tap water were applied. The results showed that the lot 1 or control (fresh seeds), lot 2 (seeds stored for 14 days) and lot 3 (seeds stored for 21 days) showed germination rates, respectively, of the order of 72.22, 55.56 and $41.67 \%$. Soaking of the seeds in the tap water significantly reduced the germination time limit of seeds from lot 1 to 25 days and increased the germination rate of pretreatment 4 of lot 1 (seeds having remained in water for 6 days) $100 \%$. This study shows to produce seedlings, fresh seeds and those dried and stored for two and three weeks can be used.

(C) 2019 International Formulae Group. All rights reserved
\end{abstract}

Keywords: Tieghemella heckelii, threatened species, storage seeds, pretreatment, germination parameters, Côte d'Ivoire.

\section{INTRODUCTION}

Dans les pays tropicaux, le développement économique est étroitement lié aux activités réalisées dans les forêts (FAO, 2003). En Côte d'Ivoire, les performances agricoles et l'exploitation forestière pour grumes, ont entrainé la disparition de plusieurs espèces de plantes dont les espèces de bois d'œuvre (Konan et al., 2005). Comme exemple, Tieghemella heckelii Pierre ex A. Chev. (Sapotaceae) ou Makoré, une espèce de bois d'œuvre de grande valeur commerciale est en voie de disparition de la flore ivoirienne. En effet, Souza Rogeria et al. (2003) indiquait que les arbres de Makoré devenaient rares même si parfois ils se rencontraient en groupe de quelques individus, dans les forêts tropicales. Dans les forêts ivoiriennes, les travaux de AkéAssi (2002) ont montré aussi que les densités moyennes de Makoré étaient de 0,1 et de 1,4 tige par hectare (diamètre supérieure à $10 \mathrm{~cm}$ ), respectivement, dans les forêts classées de Mopri et d'Irobo. Or, la régénération naturelle de l'espèce est jugée faible par des auteurs, tels qu'Ettien (2005) dans la forêt classée du Haut Sassandra, Kouadio (2007) dans la forêt classée de la Bossematié. C'est donc à juste titre que l'UICN (2018) qualifie le Makoré d'une espèce en danger d'extinction dans les forêts tropicales. Par ailleurs, selon Kouadio et al. (2007), la dissémination des graines de Makoré était principalement assurée par l'éléphant (Loxodonta cyclotis), ci-bien que la diminution des populations d'éléphants pourrait compromettre la régénération de l'espèce. Mais des études récentes réalisées sur la domestication de Makoré par Bonnéhin (2000), dans la zone périphérique Ouest du Parc National de Taï (PNT), en Côte d'Ivoire, ont montré que les graines fraîches présentent un fort taux de germination, de l'ordre de $90 \%$. Or, pour la préservation et la pérennisation de l'espèce, il est nécessaire de conserver les semences. Cependant, la question est de savoir si la conservation des graines influencerait leur capacité de germination. D'où l'intérêt de cette étude qui a pour but de collecter des informations sur les paramètres de germination (délai, durée et taux) des graines conservées de Makoré.

L'objectif général de cette étude est de disposer des données sur le potentiel de germination des graines conservées de Makoré. Spécifiquement, il s'agit de:

- Déterminer le délai de germination des différents lots de grains conservées: 
- Déterminer la durée de germination des différents lots de graines conservées;

- Déterminer le taux de germination des différents lots de graines conservées.

\section{MATERIEL ET METHODES Matériel}

L'étude a été réalisée dans l'arboretum du Centre National de Floristique (CNF), situé au sein de 1'Université Félix HouphouëtBoigny (UFHB), dans le District Autonome d'Abidjan, en Côte d'Ivoire (Figure 1). Situé dans le secteur ombrophile du domaine guinéen, le District autonome d'Abidjan est caractérisé par une température moyenne annuelle de l'ordre $26{ }^{\circ} \mathrm{C}$ et par une pluviométrie moyenne annuelle de l'ordre de 1658,64 mm. Les sols du District Autonome d'Abidjan, incluant ceux du CNF sont pour la plupart ferralitiques et fortement désaturés sous forte pluviométrie (Ouattara, 2017). La végétation du $\mathrm{CNF}$ est du type forêt dense sempervirente. Cette végétation n'est pas naturelle, elle est du fait de l'homme par l'introduction de nombreuses espèces à majorité forestière dans les 11 parcelles de l'arboretum (Doumbia, 2014). La création des parcelles n'a pas été définie, en fonction de thématiques spécifiques. De ce fait, les espèces ont été introduites de façon aléatoire.

Le matériel biologique utilisé au cours de cette étude est constitué de graines de Makoré. L'un des géants des forêts denses humides sempervirentes tropicales, il porte un fût assez droit, les feuilles sont simples subalternes, lâchement onduleuses, groupées au sommet des rameaux. Les fruits sont des baies ovoïdes, de couleur jaune-verdâtre à maturité fraiches (Figure 2). Pour le test de germination, les graines fraiches et les graines séchées et conservées ont été utilisées (Figure 3).

\section{Méthodes d'étude \\ Collecte de données}

Sélection de semences et constitution des lots de graine

Les fruits matures ont été ramassés et sélectionnés, en pleine période de fructification, sous les semenciers de Makoré, dans l'arboretum du Centre National de Floristique (CNF). Les fruits ont été d'abord soigneusement débarrassés de leurs pulpes; ensuite, les graines obtenues ont été plongées dans une bassine remplie d'eau de robinet pendant 10 minutes; celles qui flottaient ont été éliminées (Figure 4). Les graines de bonne qualité recueillies au fond de la bassine ont été séchées en les étalant sur les paillasses, à température ambiante, dans l'ombrière du CNF, pendant 7 jours. Après le séchage, la constitution des différents lots de graines a été effectuée, selon la durée de conservation, avant le semis. Cinq lots ont été constitués et répartis comme suit : le lot 1 ou témoin composé de graines fraiches ; le lot 2 comprenant les graines séchées et conservées pendant 14 jours ; le lot 3 renfermant les graines séchées et conservées pendant 21 jours ; le lot 4 constitué de graines séchées et conservées pendant 97 jours et le lot 5 composé de graines séchées et conservées pendant 187 jours. Chaque lot est composé de 36 graines, soit un total de 180 graines utilisées, pour la réalisation de cette étude.

\section{Prétraitement des différents lots de graines de Makoré}

Les graines des 5 lots constitués ont fait l'objet de prétraitement pour ramollir les téguments qui sont coriaces. Pour ce faire, les 36 graines de chaque lot ont séjourné pendant 1 à 6 jours dans l'eau de robinet. Pour chacun des lots, les 36 graines ont été subdivisées en 4 sous-lots (échantillons) de 9 graines chacun. Tous les échantillons ont subi 4 types de traitements (Tableau 1).

\section{Dispositif expérimental et suivi de l'essai de germination de Makoré}

Le dispositif expérimental est constitué de 20 planches de semis, disposées en 5 colonnes pour les 5 lots de graines et 4 lignes pour les 4 traitements. Les planches sont séparées par des allées de 0,5 mètres de largeur, chacune, pour permettre une bonne aération et l'arrosage des semis. Chaque planche de semis est composée de 3 rangés de 3 sachets de pépinières, disposés les uns à côté des autres (Figure 5). Les sachets en polyéthylène de 30 
$\mathrm{cm}$ de longueur et de $26 \mathrm{~cm}$ de largueur, au nombre de 180 , sont remplis de terre prélevée dans le sous-bois de l'arboretum du CNF. Une graine a été semée, dans chaque sachet, soit un total de 180 graines saines utilisées. Durant les 60 jours d'expérience, la pépinière a été arrosée 2 fois par jour (à 8 h et à $16 \mathrm{~h}$ ), durant les 2 premières semaines, puis un seul arrosage tous les jours à $8 \mathrm{~h}$, jusqu'à la fin de l'expérience.

\section{Collecte des données sur l'essai de germination des graines de Makoré}

Pour les différents paramètres de germination déterminés à savoir le délai, la durée et le taux, les mesures ont été réalisée dès le lendemain des semis et s'est étendue sur une période de 60 jours. Pour chaque lot et traitement, le nombre de jours entre la date de semis et celle de la ou des première (s) graine (s) germée (s) a été noté, pour le délai de germination. Pour la détermination de la durée de germination, le nombre de jours entre la date des premières et celle des dernières graines germées, a été noté. Concernant le taux de germination, les graines germées, dans chacun des 20 planches de semis, ont été dénombrées.

\section{Analyse des données}

Paramètres de germination des graines de Makoré

Les valeurs moyennes du délai de germination, de la durée de germination et du taux de germination par lot et par traitement ont été calculées et analysées statistiquement. Pour le taux de germination (TG), les valeurs ont été calculées à partir de l'expression mathématique suivante :

$$
T G(\%)=\frac{n}{N} \times 100
$$

Avec $n$ : nombre de graines germées et $N$ : nombre total de graines semées.

\section{Analyse statistique}

Les variations des paramètres de germination (délai de germination, durée de germination et taux de germination) des différents lots de graines ont été analysées à partir de l'analyse de variance (ANOVA). Le test de Newman Keuls a été utilisé pour le classement des moyennes avec un seuil de probabilité $(\alpha=5 \%)$, à l'aide du logiciel Statistica 7.1.
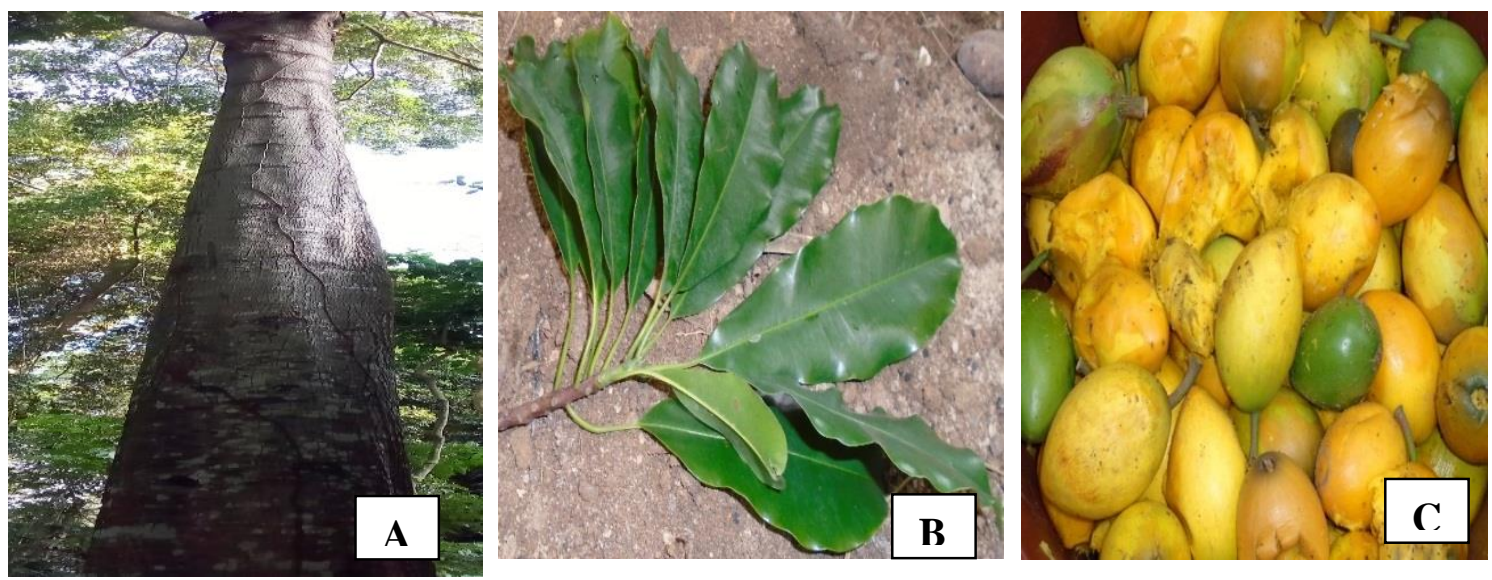

Figure 2 : Aperçu de différents organes d'un Makoré.

A : Fût observé dans l'arboretum du Centre National de Floristique (CNF)

$\mathrm{B}$ : Rameau feuillé récolté dans l'arboretum du CNF

C : Fruits matures (jaune-verdâtre) et non matures (vert) collectés sous les semenciers de Makoré dans l'arboretum du CNF. 

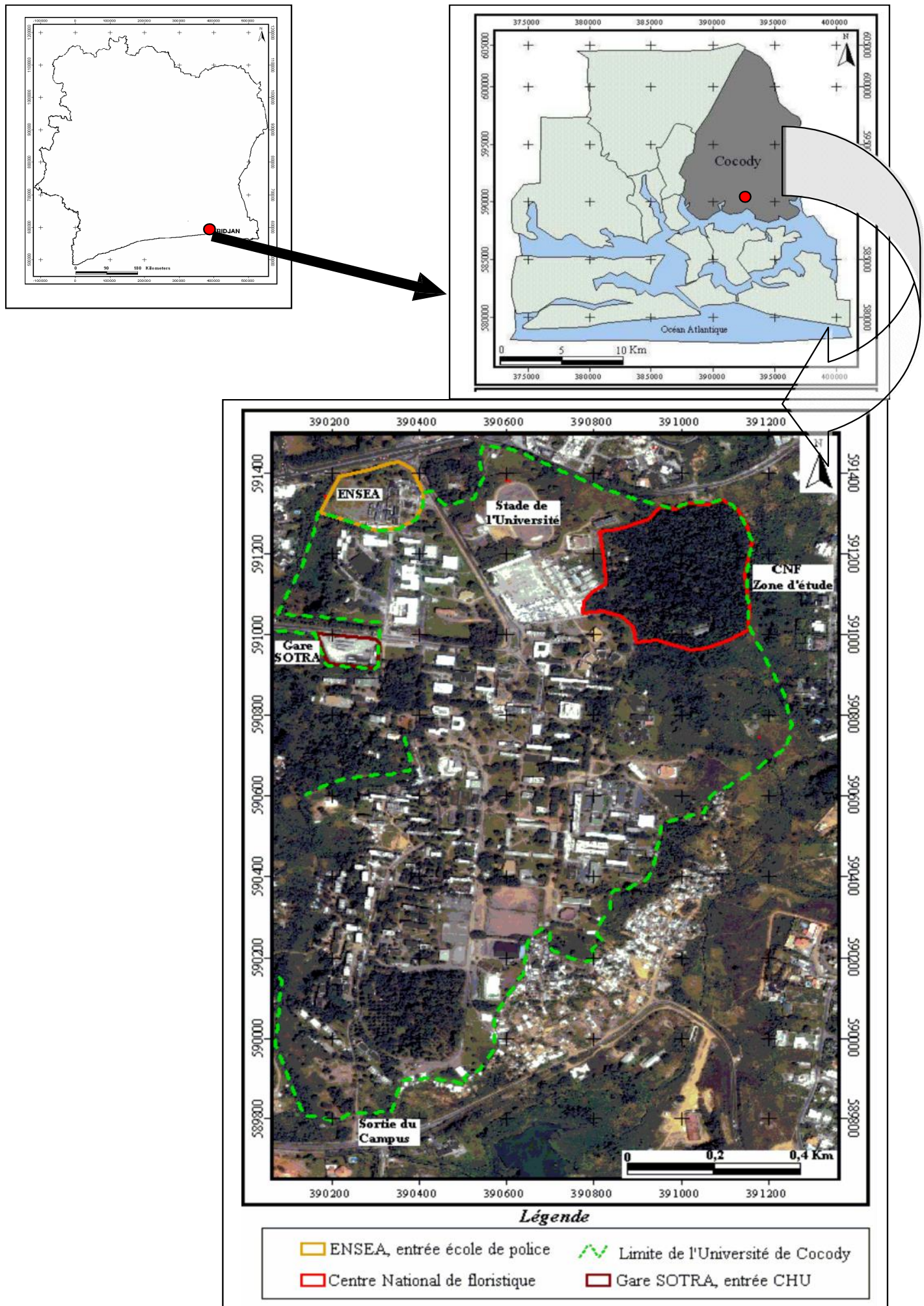

Figure 1: Localisation du Centre National de Floristique (CNF) de l'Université Félix Houphouët Boigny dans le District Autonome d'Abidjan.

(Source: Ouattara, 2017) 

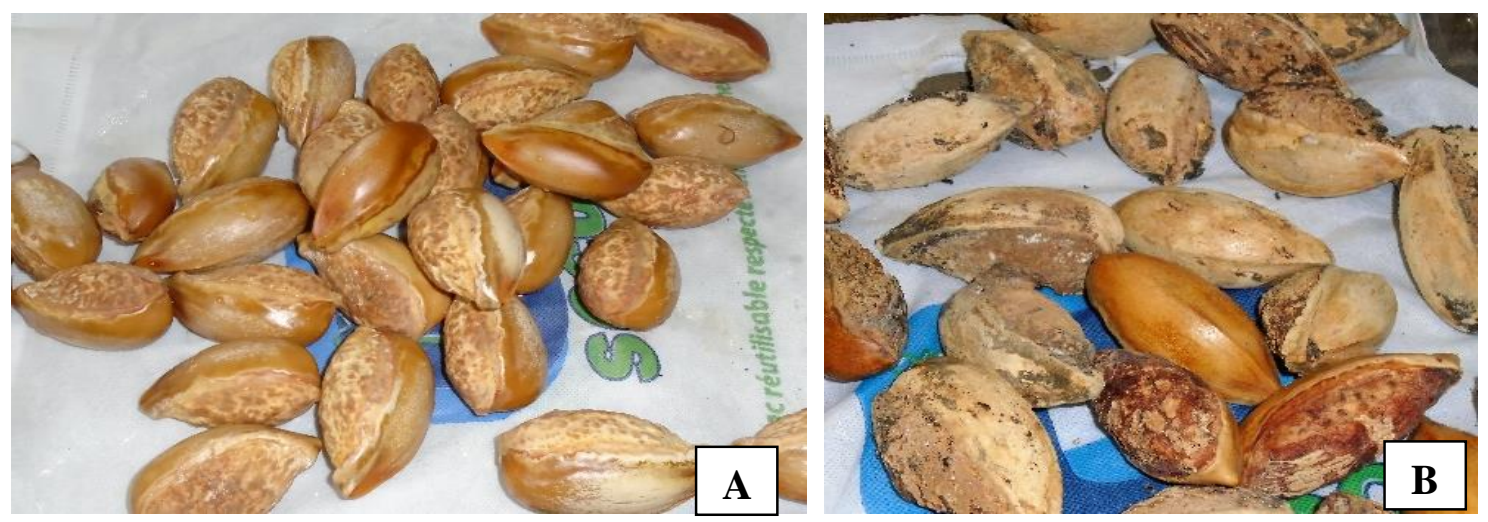

Figure 3 : Aperçu de différents types de graines de Makoré.

A : Graines fraiches

B : Graines séchées

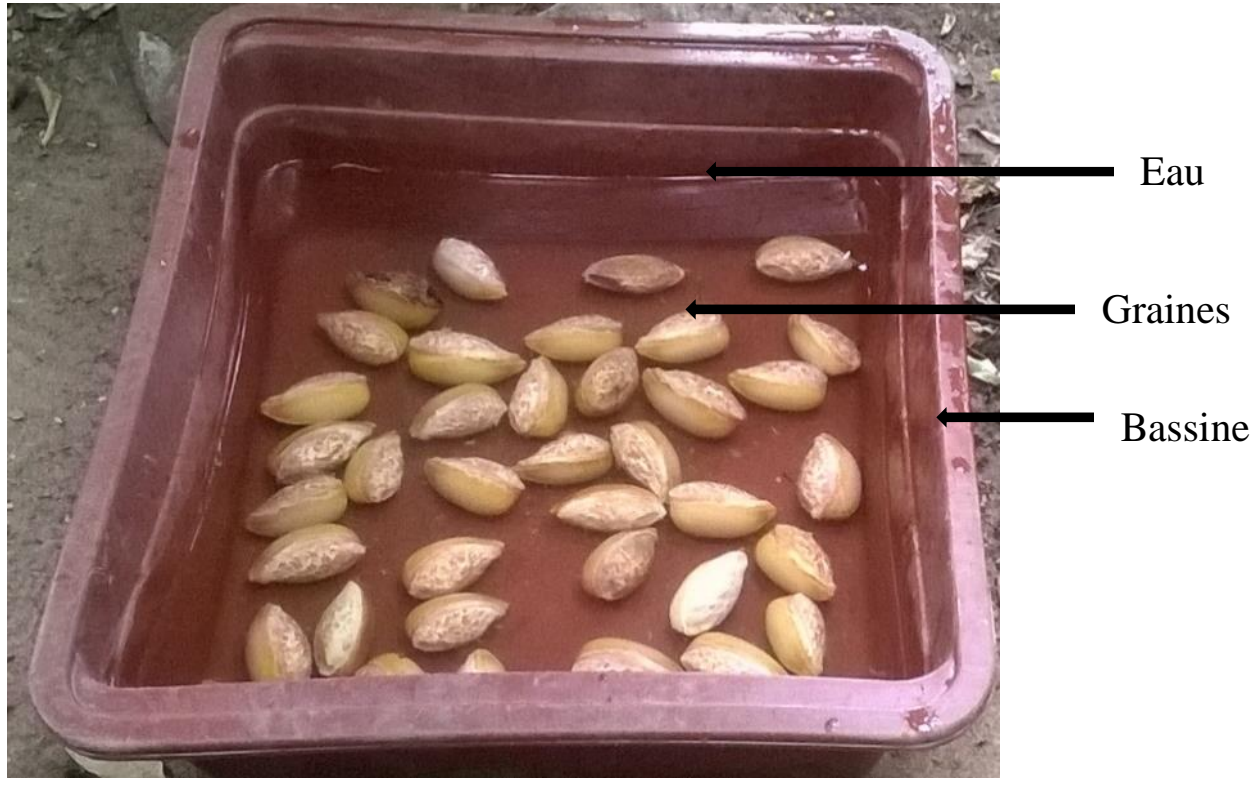

Figure 4 : Aperçu de la technique de contrôle de la qualité des graines de Makoré par trempage dans l'eau de robinet.

Tableau 1 : Traitement à l'eau des lots de graines de Makoré.

\begin{tabular}{clllll}
\hline & \multicolumn{5}{c}{ Traitements (T) } \\
\cline { 2 - 6 } Lots & T1 & \multicolumn{2}{c}{ T2 } & \multicolumn{1}{c}{ T3 } & \multicolumn{1}{c}{ T4 } \\
\hline Lot 1 & $\begin{array}{l}\text { Neuf graines non } \\
\text { trempées dans l'eau }\end{array}$ & $\begin{array}{l}\text { Neuf grains trempées } \\
\text { dans l'eau pendant 1 } \\
\text { jour }\end{array}$ & $\begin{array}{l}\text { Neuf } \\
\text { trempées dans l'eau } \\
\text { pendant 3 jours }\end{array}$ & $\begin{array}{l}\text { Neuf } \\
\text { trempées dans l'eau } \\
\text { pendant 6 jours }\end{array}$ \\
Lot 2 & $\begin{array}{l}\text { Neuf graines non } \\
\text { trempées dans l'eau }\end{array}$ & $\begin{array}{l}\text { Neuf grains trempées } \\
\text { dans l'eau pendant 1 } \\
\text { jour }\end{array}$ & $\begin{array}{l}\text { Neuf } \\
\text { trempées dans l'eau } \\
\text { pendant 3 jours }\end{array}$ & $\begin{array}{l}\text { Neuf } \\
\text { trempées dans l'eau } \\
\text { pendant 6 jours }\end{array}$
\end{tabular}


Lot 3 Neuf graines non trempées dans l'eau

Lot 4 Neuf graines non trempées dans l'eau

Lot 5 Neuf graines non trempées dans l'eau
Neuf grains trempées Neuf grains dans l'eau pendant 1 trempées dans l'eau jour

Neuf grains trempées Neuf grains dans l'eau pendant 1 trempées dans l'eau jour

Neuf grains trempées Neuf grains dans l'eau pendant 1 trempées dans l'eau jour
Neuf

grains trempées dans l'eau pendant 6 jours

Neuf grains trempées dans l'eau pendant 6 jours

Neuf grains trempées dans l'eau pendant 6 jours

1
Trt 12
3

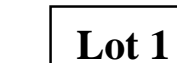

\section{Lot 2}

\section{Lot 3}

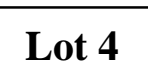

\section{Lot 5}

Trt 2

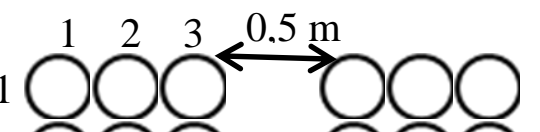
3
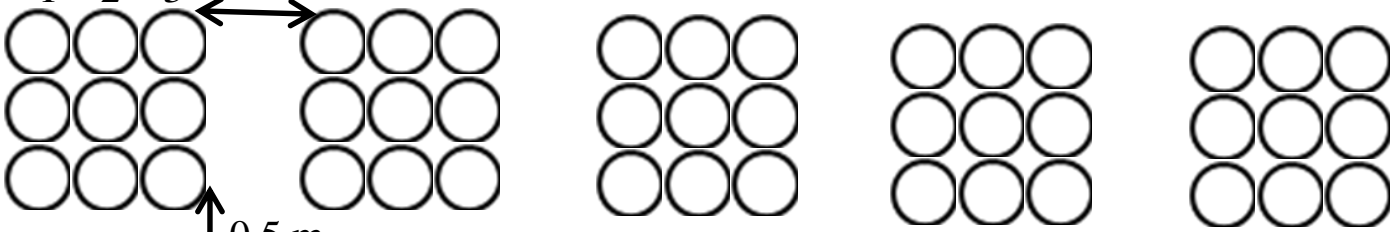
$0,5 \mathrm{~m}$

Trt 3
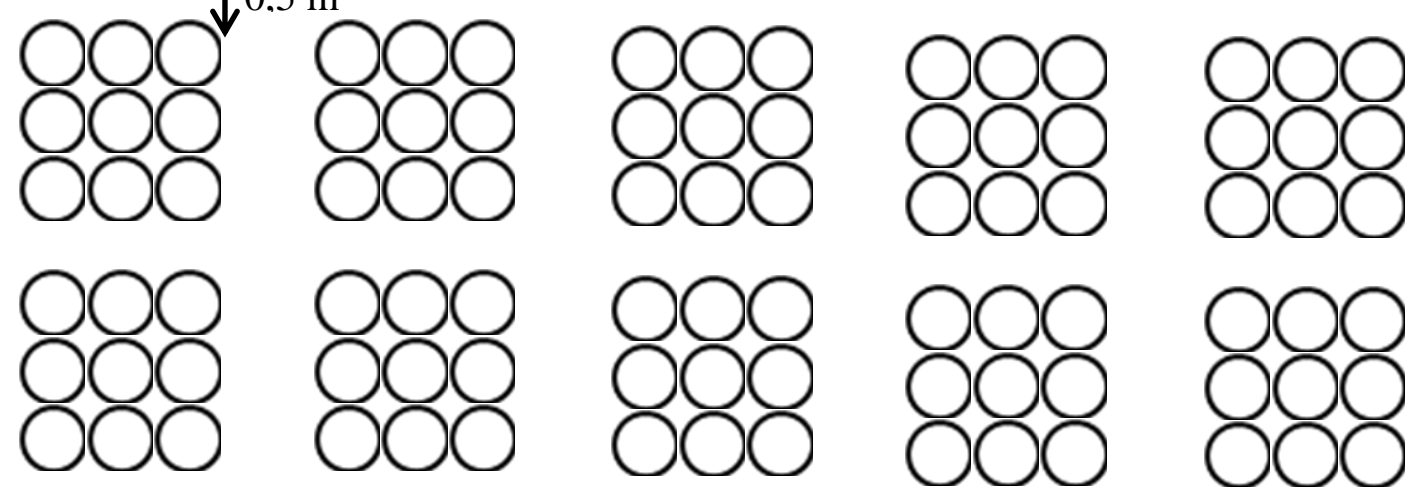

Trt 4
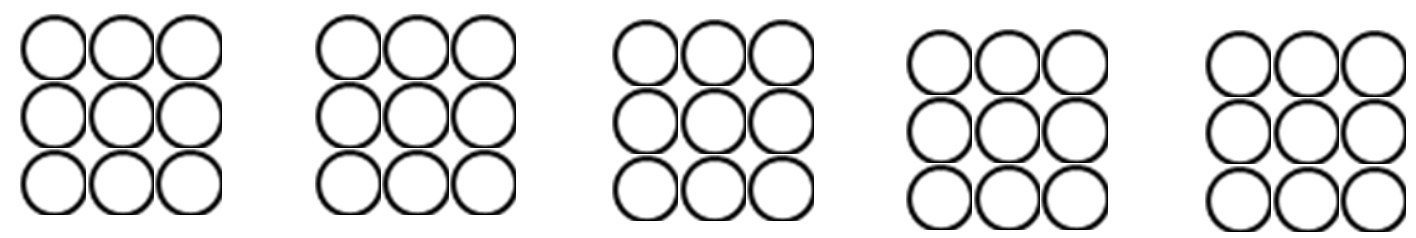

: Sachets dans lesquels les graines ont été semées

$\longleftrightarrow$ : Allées de séparation entre les planches de semis de $0,5 \mathrm{~m}$ de largeur

Lot $1:$ Graines fraiches

Lot 2 : Graines séchées et conservées, pendant 1 semaine

Lot 3 : Graines séchées et conservées, pendant 2 semaines

Lot 4 : Graines séchées et conservées, pendant 3 mois

Lot 5 : Graines séchées et conservées, pendant 6 mois

Trt 1 : Traitement 1 (Semis sans trempage)

Trt 2 ; Traitement 2 (Semis par trempage, pendant 1 jour)

Trt 3 ; Traitement 2 (Semis par trempage, pendant 3 jours)

Trt 4 ; Traitement 4 (Semis par trempage, pendant 6 jours)

Figure 5: Schéma du dispositif expérimental de l'essai de germination. 


\section{RESULTATS}

\section{Délai et durée de germination des différents} lots de graines en fonction des traitements et des durées de conservation

L'analyse des résultats du Tableau 2 ont montré que sur les 5 lots de graines testés, seuls les lots 4 et 5 n'ont pu germer, au terme des 60 jours d'expérience. Par contre, les graines des lots 1,2 et 3 ont présenté un délai de germination qui varie de 25,33 à 48,00 jours, en fonction de la durée de conservation de celles-ci. Les graines fraiches du lot 1 (témoin) ont eu un délai de germination court de l'ordre de 25 jours par rapport aux lots 2 et 3 qui ont enregistré un délai relativement long. Pour les graines de ces 2 lots, il fallait attendre plus d'un mois, respectivement 32 et 35 jours pour observer la sortie de la plantule. Contrairement aux résultats du délai de germination, les graines déshydratées et conservées des lots 2 et 3 ont eu une durée de germination relativement courte. Les graines du lot 3 ont une germination groupée (8 à 10 jours), celle des graines du lot 2 est moyennement étalée ( 8 à 17 jours) alors que les graines du lot 1 présentent une germination dispersée (4 à 20 jours).

Le trempage des graines dans l'eau de robinet a raccourci le délai de germination ( 25 jours pour le traitement 4 ), cependant, il a eu un effet mitigé sur la durée de germination. Les résultats de l'analyse statistique consignés dans le Tableau 2 ont montré qu'il existe une différence significative, seulement, entre les valeurs moyennes de la durée de germination des graines traitées et celles non traitées du lot $1(\mathrm{~F}=15,83 ; \mathrm{P}=0,001)$.
Variation des taux de germination des différents lots de graines en fonction des traitements et des durées de conservation

Les taux de germination des 3 lots de graines germées varient en fonction des traitements et des durées de conservation. D'une manière générale, le taux de germination des graines semées a été faible, soit 33, $89 \%$ (Tableau 3).

Pour l'effet des durées de conservation des graines sur leur germination, l'analyse du Tableau 3 a montré que les graines fraiches du lot 1 ont présenté un taux de germination relativement élevés, de l'ordre de 72,22\% par rapport aux graines séchées et conservées qui ont enregistré des taux moyens, respectivement de l'ordre de 55, 56\% pour le lot 2 et $41,67 \%$ pour le lot 3. Pour l'effet du trempage des graines sur la germination, la Figure 6 montre que les valeurs du taux de germination des différents traitements des graines fraiches du lot 1 sont croissantes; la valeur du taux de germination du traitement 4 est sensiblement le double de celle des traitements 1,2 et 3 . Pour les lots 2 et 3 , les forts taux de germination des graines ont été observés au niveau des traitements 1 et 4 (Figure 6). Concernant les valeurs statistiques, les résultats consignés dans le Tableau 4 ont montré qu'il existe une différence significative entre les valeurs moyennes du taux de germination des graines des différents traitements du lot $1(\mathrm{~F}=6,56$; $\mathrm{P}=0,002)$. Pour les lots 2 et 3 , les valeurs du taux moyens de germination des traitements du lot 2 sont statistiquement différentes $(\mathrm{F}=6,2$; $\mathrm{P}=0,02$ ) alors qu'aucune différence significative n'a été observée entre les valeurs moyennes du taux de germination des traitements du lot $3(\mathrm{~F}=0,69 ; \mathrm{P}=0,58)$. 
Tableau 2 : Comparaison des valeurs moyennes des délais (TL) et des durées (DG) de germination des graines de Makoré en fonction des traitements et des lots.

\begin{tabular}{llllllll}
\multirow{2}{*}{ Lots } & PG & \multicolumn{5}{c}{ Traitements } & \\
\cline { 3 - 7 } & & Traitement 1 & Traitement 2 & Traitement 3 & Traitement 4 & \\
\hline \multirow{2}{*}{ Lot 1 } & TL (jour) & $41,67 \pm 9,24^{\mathrm{a}}$ & $31,00 \pm 0,57^{\mathrm{a}}$ & $26,66 \pm 1,45^{\mathrm{a}}$ & $25,33 \pm 1,67^{\mathrm{a}}$ & $\mathrm{F}=2,42 ; \mathrm{p}=0,14$ \\
& DG (jour) & $4,67 \pm 1,45^{\mathrm{c}}$ & $6,67 \pm 2,60^{\mathrm{c}}$ & $14,00 \pm 1,15^{\mathrm{b}}$ & $20,33 \pm 1,67^{\mathrm{a}}$ & $\mathrm{F}=15,83 ; \mathrm{p}=0,001$ \\
\hline \multirow{2}{*}{ Lot 2 } & TL (jour) & $40,33 \pm 1,20^{\mathrm{ab}}$ & $42,67 \pm 2,73^{\mathrm{a}}$ & $39,00 \pm 3,21^{\mathrm{ab}}$ & $32,00 \pm 1,00^{\mathrm{b}}$ & $\mathrm{F}=4,17 ; \mathrm{p}=0,08$ \\
& DG (jour) & $11,67 \pm 1,33^{\mathrm{a}}$ & $17,67 \pm 1,33^{\mathrm{a}}$ & $11,67 \pm 1,45^{\mathrm{a}}$ & $8,00 \pm 4,04^{\mathrm{a}}$ & $\mathrm{F}=2,91 ; \mathrm{p}=0,10$ \\
\hline \multirow{2}{*}{ Lot 3 } & TL (jour) & $42,00 \pm 3,51^{\mathrm{a}}$ & $42,00 \pm 3,51^{\mathrm{a}}$ & $35,02 \pm 2,00^{\mathrm{a}}$ & $48,00 \pm 4,00^{\mathrm{a}}$ & $\mathrm{F}=2,53 ; \mathrm{p}=0,13$ \\
& DG (jour) & $8,00 \pm 2,08^{\mathrm{a}}$ & $9,33 \pm 2,03^{\mathrm{a}}$ & $10,00 \pm 4,04^{\mathrm{a}}$ & $8,00 \pm 3,79^{\mathrm{a}}$ & $\mathrm{F}=0,10 ; \mathrm{p}=0,96$ \\
\hline \multirow{2}{*}{ Lot 4 } & TL (jour) & 0 & & 0 & 0 & 0 & 0 \\
& DG (jour) & 0 & 0 & 0 & 0 & 0 \\
\hline \multirow{2}{*}{ Lot 5 } & TL (jour) & 0 & & 0 & 0 & 0 & 0 \\
& DG (jour) & 0 & 0 & 0 & 0 & 0 \\
\hline
\end{tabular}

PG : Paramètre de germination, F : Test de décision de Ficher, P : Probabilité $(\alpha=5 \%)$.

Les moyennes sur les lignes accompagnées des mêmes lettres ne sont pas significativement différentes au seuil de $\alpha=5 \%$.

Tableau 3 : Nombre de graines de Makoré germées par traitements et par lots.

\begin{tabular}{lcccccc}
\hline \multicolumn{1}{c}{ Traitements } & \multicolumn{5}{c}{ Lots } & \multirow{2}{*}{ Total } \\
\cline { 2 - 5 } Traitement 1 & 5 & 8 & 6 & 0 & 0 & 19 \\
Traitement 2 & 5 & 4 & 3 & 0 & 0 & 12 \\
Traitement 3 & 7 & 3 & 2 & 0 & 0 & 12 \\
Traitement 4 & 9 & 5 & 4 & 0 & 0 & 18 \\
\hline $\begin{array}{l}\text { Total } \\
\text { Taux de }\end{array}$ & 26 & 20 & 15 & 0 & 0 & 61 \\
\hline $\begin{array}{l}\text { Termination }(\%) \\
\text { T2, 22 }\end{array}$ & $\mathbf{5 5 , 5 6}$ & $\mathbf{4 1 , 6 7}$ & $\mathbf{0}$ & $\mathbf{0}$ & $\mathbf{3 3 , 8 9}$ \\
\hline
\end{tabular}




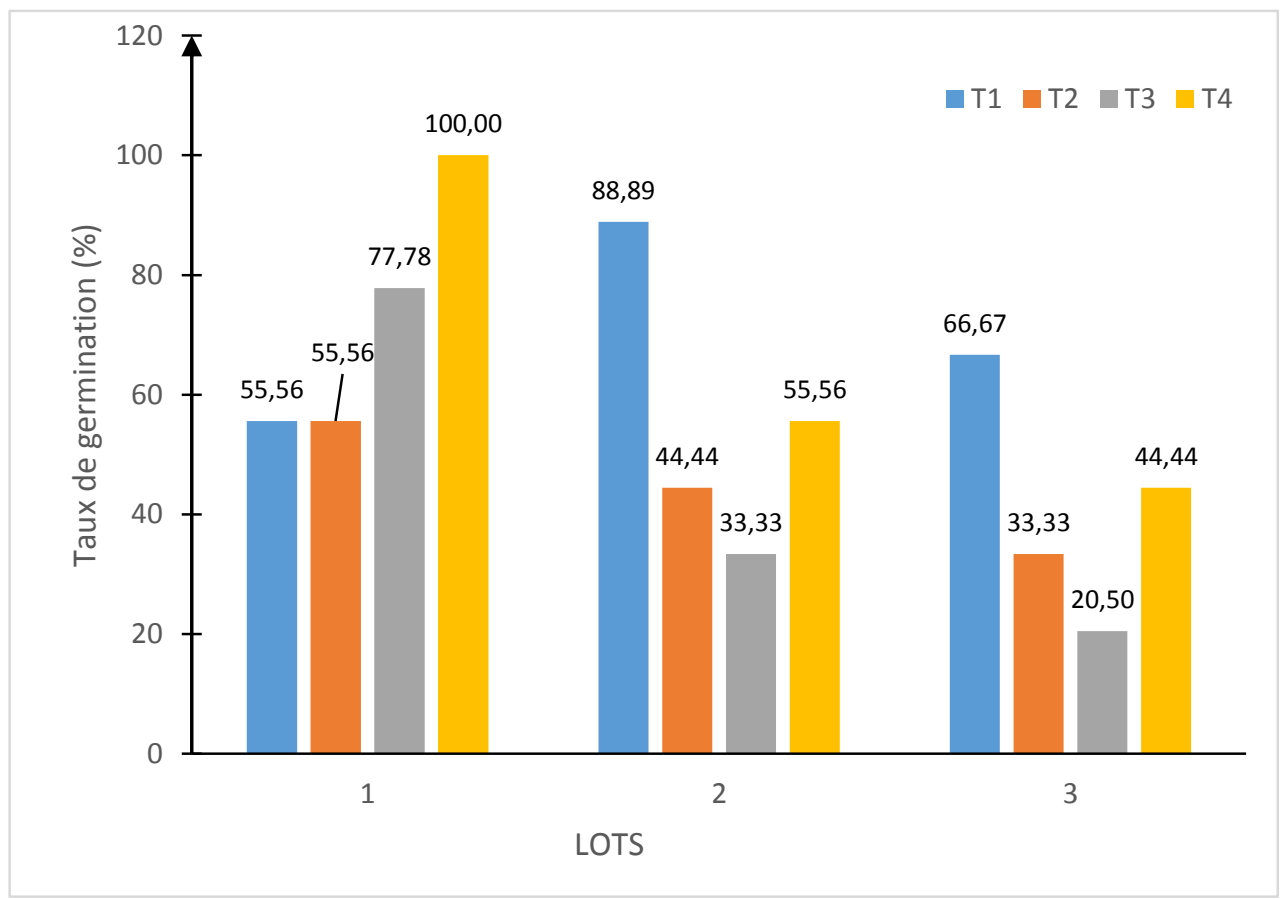

T1 : Traitement $1 \quad \mathrm{~T} 2:$ Traitement $2 \quad \mathrm{~T} 3:$ Traitement $3 \quad \mathrm{~T} 4:$ Traitement 4

Figure 6: Évolution des taux de germination des trois lots de graines germés de Makoré en fonction des traitements et des durées de conservation.

Tableau 4: Comparaison des valeurs moyennes du taux de germination des grains de Makoré par traitements et par lots.

\begin{tabular}{ccccccc}
\hline & \multicolumn{5}{c}{ Traitements (T) } & \multicolumn{2}{c}{ Valeurs statistiques } \\
\cline { 2 - 5 } Lots & T1 & T2 & T3 & T4 & \\
\hline Lot 1 & $1,67 \pm 0,33^{\mathrm{b}}$ & $1,67 \pm 0,33^{\mathrm{b}}$ & $1,33 \pm 0,33^{\mathrm{b}}$ & $3,00 \pm 0,00^{\mathrm{a}}$ & $\mathrm{F}=6,56 ;$ & $\mathrm{P}=0,02$ \\
Lot 2 & $2,67 \pm 0,33^{\mathrm{a}}$ & $1,33 \pm 0,33^{\mathrm{b}}$ & $1,00 \pm 0,00^{\mathrm{b}}$ & $1,67 \pm 0,33^{\mathrm{b}}$ & $\mathrm{F}=6,2 ;$ & $\mathrm{p}=0,02$ \\
& & & & & & \\
Lot 3 & $2,00 \pm 0,00^{\mathrm{a}}$ & $1,00 \pm 0,58^{\mathrm{a}}$ & $2,00 \pm 1,00^{\mathrm{a}}$ & $1,33 \pm 0,33^{\mathrm{a}}$ & $\mathrm{F}=0,69 ;$ & $\mathrm{p}=0,58$ \\
Lot 4 & 0 & 0 & 0 & 0 & 0 & 0 \\
Lot 5 & 0 & 0 & 0 & 0 & 0 & 0 \\
\hline
\end{tabular}

F : Test de décision de Ficher, $\mathrm{P}$ : Probabilité $(\alpha=5 \%)$.

Les moyennes sur les lignes accompagnées des mêmes lettres ne sont pas significativement différentes au seuil de $\alpha=5 \%$. 


\section{DISCUSSION}

L'absence de germination des graines des lots 4 et 5 , au terme des 60 jours d'expérience, permet de qualifier les semences de Makoré de récalcitrantes. En effet, celles-ci pourraient être exigeantes en eau, donc moins tolérantes à la dessiccation; c'est à dire que plus les graines sont conservées plus, elles perdent leur viabilité. De fait, les graines des lots 4 et 5 , ont été séchées pendant 7 jours et conservées, respectivement pendant 3 et 6 mois, avant le semis. Cette longue conservation a entraîné, probablement, la perte de leur pouvoir germinatif. D'ailleurs, les graines des lots 2 et 3 , séchées pendant 7 jours et conservées, respectivement pendant une semaine et 2 semaines, ont vu leur potentialité germinative baissée par rapport aux graines fraiches. En fait, les graines de Makoré renferment de la matière grasse; elles supportent donc difficilement le séchage et la longue conservation. C'est pourquoi, les auteurs tels que Gourlet-Fleury (2002), Loth et al. (2005), Traoré et al. (2005), Ouédraogo et al. (2006) et Dan Guimbo et al. (2010) ont indiqué que les semences forestières renfermant de la matière grasse ne peuvent se conservées pendant plus d'un mois. Par contre, les graines fraiches du lot 1 ont montré de bonnes capacités de germination. Quoique l'expérience ait été réalisée sous une intensité d'ensoleillement modérée, les résultats de cette étude sont conformes à ceux obtenus par Bonnéhin (2000) qui a souligné que les graines fraiches de Makoré présentent de forte potentialité germinative, de l'ordre de $90 \%$, en milieu ouvert.

Le trempage des graines de Makoré dans l'eau de robinet a eu un effet positif sur leur germination. L'action de l'eau a réduit considérablement le délai de germination qui est de 25 jours, pour les graines fraiches, au cours de cette étude, alors que les résultats des travaux de Bonnéhin (2000) indiquaient 4 à 5 semaines de germination, après le semis. En effet, le trempage des graines a entrainé l'imbibition (ramollissement) rapide du tégument et l'entrée d'eau dans les réserves qui a permis le déclenchement des réactions métaboliques de l'embryon favorisant ainsi la sortie rapide de la plantule, comme Ahoton et al. (2009) l'ont réalisé sur les semences de Prosopis africana (Gill., Perrot. et Rich.) Taub. (Fabaceae). Le délai de germination relativement long pour les graines séchées et conservées, malgré le trempage, pourrait s'expliquer par le fait que le tégument soit devenu plus coriace avec la conservation. D'où l'imbibition de celui-ci s'est réalisée lentement et a rendu difficile l'entrée d'eau dans les réserves des graines, retardant ainsi l'émergence de la plantule.

\section{Conclusion}

Cette étude a pour objectif de maitriser les paramètres de germination des graines de Makoré, afin de l'utiliser dans les reboisements des forêts classées en Côte d'Ivoire, en vue de sa pérennisation. Elle a montré qu'au terme des 60 jours d'expérience, la variation des valeurs du délai, de la durée et du taux de germination est étroitement liée aux durées de conservation et au traitement à l'eau des graines de Makoré. Les graines déshydratées et conservées pendant longtemps, au-delà de trois mois, n'ont pu germées, alors que les graines fraiches et celles conservées pendant deux et trois semaines ont présenté des potentialités de germination assez remarquable. Le trempage des graines dans l'eau de robinet a influencé positivement le délai et le taux de germination, mais il a eu un effet mitigé sur la durée de germination. Il ressort de cette étude que plus les graines sont conservées pendant longtemps, à température ambiante, plus leur capacité de germination est faible et devient nulle, au-delà, de trois mois.

$\mathrm{Au}$ regard de ce qui précède, il est souhaitable, pour le reboisement des forêts tropicales, que les graines fraiches de Makoré qui possèdent de bonnes potentialités de germination soient utilisées pour la production de plants. Aussi les graines séchées et conservées jusqu'à trois semaines peuventêtre, également, utilisées. 


\section{CONFLIT D'INTERETS}

Nous déclarons n'avoir aucun conflit d'intérêts.

\section{CONTRIBUTIONS DES AUTEURS}

$\checkmark$ KK est l'auteur principal de cet article, a défini le thème et les objectifs de cette étude. Il a orienté la demarche méthodologique et les résultats obtenus. Il a participé à la redaction du manuscrit.

$\checkmark$ SS a contribué à la définition de la démarche méthodologique et les résultats obtenus. Il a aussi supervisé la collecte des données sur le terrain, participé aux analyses de données et à la rédaction du manuscrit.

$\checkmark$ EKK a contribué significativement à la rédaction de cet article de publication en corrigeant la forme et le fond du manuscrit.

$\checkmark$ SCD a collecté les données sur le terrain, réalisé les différentes analyses et rédigé le mémoire de son Master en Biologie Végétale, avec les résultats obtenus de cette étude.

\section{REMERCIEMENTS}

Nous exprimons notre reconnaissance au Directeur du Centre National de Floristique de l'Université Félix HOUPHOUËT-BOIGNY (Côte d'Ivoire) de nous avoir permis de collecter les semences de Makoré dans l'arboretum et de réaliser nos travaux dans l'ombrière dudit centre.

Nous disons aussi merci au Directeur du Jardin Botanique de Bingerville (Côte d'Ivoire) de nous avoir autorisés de collecter également les semences de Makoré dans le Jardin.

\section{REFERENCES}

Aké-Assi L. 2002. Flore de la Côte d'Ivoire 2: Catalogue, Systématique, Biogéographie et Ecologie. Boissera 58 : Suisse.

Ahoton LE, Adjakpa JB, M'po IM, Akpo EL. 2009. Effet des prétraitements des semences sur la germination de Prosopis africana (Guill., Perrot. et Rich.) Taub. (Fabaceae). Tropicultura, 27(4): 233238.

DOI : http://tropicultura.org/text/v27n4/233.pdf

Bonnéhin L. 2000. Domestication paysanne des arbres fruitiers forestiers: cas de Coula edulis Baill. Olacaceae et de Tieghemella heckelii Pierre ex A. Chev., Sapotaceae, autour du Parc national de Taï, Tropenbos-Côte d'Ivoire. Abidjan, Côte d'Ivoire, p. 129.

Dan Guimbo I, Mahamane A, Ambouta KJM. 2010. Peuplement des parcs à Neocarya macrophylla (Sabine) Prance et à Vitellaria paradoxa (Gaertn. C.F.) dans le Sud-Ouest Nigérian : diversité, structure et régénération. Int. J. Biol. Chem. Sc., 4(5): $\quad$ 1706-1720. DOI: http://dx.doi.org/10.4314/ijbcs.v4i5.32

Ettien DT. 2005. Potentiels de régénération des essences forestières commerciales par la germination des graines, dans la forêt classée du Haut-Sassandra (Centre-Ouest de la Côte-d'Ivoire). Thèse de Doctorat 3ème cycle, Université Abidjan-Cocody, Abidjan, Côte d'Ivoire, $259 \mathrm{p}$.

FAO. 2003. State of the World's forests. FAO, Italy, $151 \mathrm{p}$.

Gourlet-Fleury. 2000. Régénération de l'Angélique en Guyane française: étude qualitative et quantitative du déterminisme de la reproduction, de l'installation et du développement des nouveaux individus dans le peuplement. Rapport ECOFOR, Guyane française, 27 p.

Kouadio K. 2007. Etudes de la flore, de la végétation et de l'impact de l'éclaircie sélective par dévitalisation, sur les essences principales de la forêt classée de Bossematié, Est de la Côte d'Ivoire. Thèse Unique, Université de CocodyAbidjan, Abidjan, Côte d'Ivoire, 192 p. 
Kouadio K, Kouassi KE, Kouamé NF, Traoré D. 2007. Impact de l'éclaircie sur la régénération naturelle des essences principales, dans la forêt classée de Bossematié (Côte d'Ivoire). Sci. Nat., 4(1): 27-35.

DOI: http://dx.doi.org/10.4314/scinat.v4i1.421 28

Loth PE, De Boer WF, Heitkönig IMA, Prins HHT. 2005. Germination strategy of the East African savanna tree Acacia tortilis. J. Trop. Ecol., 21(5): 509-517. DOI: https://doi.org/10.1017/50266467405002 $52 \mathrm{X}$

Ouattara SFP. 2017. Diversité et densité des espèces de bois d'œuvre inscrites sur la liste rouge de l'UICN, dans l'arboretum du Centre National de Floristique de l'Université Félix Houphouët-Boigny d'Abidjan (Côte d'Ivoire). CNF, Université Félix Houphouët-Boigny, Abidjan, Côte d'Ivoire, 60 p.
Ouédraogo A, Thiombiano A, Hahn-hadjali, Guinko S. 2006. Régénération sexuée de Boswellia dalzielii Hutch.: un arbre médicinal de grande valeur au BurkinaFaso. Bois et Forêts des Tropiques, 289(3): 41-52. DOI : http://doi.org/10.19182/bft2006.289.a20 307.

Souza Rogeria P, Ivany FM, Valio. 2003. Seedling growth of fifteen brazilian tropical tree species differing in successional status. Revista. Brasil. Bot., 26(1): $\quad 35-47 . \quad$ DOI: http://dx.doi.org/10.1590/S010084042003000100005

UICN. 2018. IUCN Red List of Threatened Species.

IUCN. http://www.iucnredlist.org. 